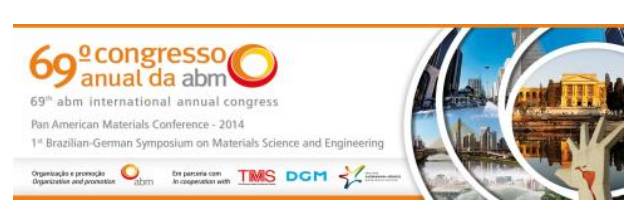

Tema: Gestão de meio ambiente e recuperação e tratamento de rejeitos

\title{
LEVANTAMENTO DAS ALTERNATIVAS DE GESTÃO DA LAMA GALVÂNICA*
}

\author{
Natália Cristina Candian Lobato ${ }^{1}$ \\ Edwin Auza Villegas² \\ Marcelo Borges Mansur ${ }^{3}$
}

\section{Resumo}

A fim de se evitar os danos causados pela corrosão, cresce cada vez mais o uso do aço galvanizado. Durante o processo de galvanização a quente ou por eletrodeposição é gerado um resíduo sólido perigoso conhecido por lama galvânica cujo descarte se trata de um processo oneroso. Sendo assim, as principais rotas de gerenciamento desse resíduo foram levantadas no presente trabalho, visando contribuir com a economia e aumento da sustentabilidade desse setor industrial. Ultimamente a lama galvânica é considerada uma matéria-prima potencial por conter metais com valor comercial presentes em concentrações elevadas, e isso tem estimulado pesquisas de rotas de recuperação desses teores metálicos. Paralelamente, a fim de resolver o problema de gestão do resíduo, estudos buscam rotas alternativas de descarte que visem estabilizar os elementos tóxicos e reutilizar a lama como matéria-prima em outro processo industrial.

Palavras-chave: Lama Galvânica; Tratamento de Resíduos; Recuperação de Metais; Meio Ambiente.

\section{A BRIEF SURVEY OF ALTERNATIVES FOR THE GALVANIC SLUDGE MANAGEMENT}

\section{Abstract}

Galvanized steel is commonly used in order to reduce damages caused by corrosion. During the galvanizing process, a hazardous waste known as galvanic sludge is generated whose disposal involves a costly operation. A survey of traditional and new treatment methods for the galvanic sludge is raised in this work, including alternative final destination possibilities. The galvanic sludge is considered a potential raw material due to its content of valuable metals normally present in high concentrations, so many studies have sought alternative treatments aiming to reuse such metals. Additionally, other studies have focused on the incorporation of the sludge in processes such as the production of cement and ceramic.

Keywords: Galvanic Sludge; Waste Treatment; Metal Recovery; Environment.

1 Engenheira Metalurgista, Mestre, Doutoranda do Programa de Pós-Graduação em Engenharia Metalúrgica, Materiais e de Minas, Universidade Federal de Minas Gerais (UFMG), Belo Horizonte, MG, Brasil.

2 Engenheiro Metalurgista, Doutor, Professor Associado, Departamento de Engenharia Metalúrgica e de Materiais, UFMG, Belo Horizonte, MG, Brasil.

3 Engenheiro Químico, Doutor, Professor Associado, Departamento de Engenharia Metalúrgica e de Materiais, UFMG, Belo Horizonte, MG, Brasil.

\footnotetext{
* Contribuição técnica ao $69^{\circ}$ Congresso Anual da ABM - Internacional e ao 14ํㅡㄹ ENEMET - Encontro Nacional de Estudantes de Engenharia Metalúrgica, de Materiais e de Minas, 21 a 25 de julho de 2014, São Paulo, SP, Brasil.
} 


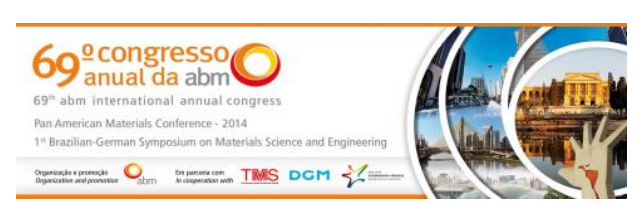

\section{INTRODUÇÃO}

O custo da corrosão para economia de um país é estimada em cerca de $4 \%$ do seu PIB para as nações industrializadas; em países emergentes esse percentual tende a ser ainda maior. Uma alternativa para minimizar esse problema é revestir o aço com outro metal empregando-se o processo de galvanização, o que tem estimulado o uso de aços galvanizados em todo o planeta [1]. Há, contudo, o potencial poluidor dos processos de recobrimento que precisa ser considerado.

Os principais processos de galvanização do aço são a quente e por eletrodeposição. Em ambos, efluentes contendo elevadas concentrações em elementos metálicos são gerados. Esses efluentes consistem dos banhos esgotados de cada etapa do processo. Seu tratamento é normalmente realizado por precipitação química, método que consiste na adição de cal hidratada ou de soda cáustica para a remoção dos metais presentes gerando uma lama galvânica, também conhecida por lodo galvânico. Estima-se que empresas de médio e grande porte produzem, em média, de 3 a 5 toneladas de lama mensalmente [2].

$\mathrm{Na}$ composição da lama galvânica estão presentes compostos químicos em diferentes formas: hidróxidos, óxidos hidratados, cloretos, géis e sais dos metais das linhas de galvanização em questão (como alumínio, cobre, cromo, níquel e zinco), além de algum gesso, sulfatos e complexos metálicos [3-5]. A composição química de uma amostra de lama galvânica do processo de recobrimento por zinco é mostrada na Tabela 1, embora se saiba que tal composição é bastante variável a depender das características do processo. Normalmente, sua classificação segundo a norma brasileira NBR 10004 [6] é como resíduo classe I - perigoso, devido à presença de metais como cádmio e cromo (principalmente na forma hexavalente), além da presença de cianeto (proveniente do processo de galvanização eletrolítica).

Tabela 1: Composição química de uma amostra de resíduo de galvanização a quente por zinco [7]

\begin{tabular}{c|c}
\hline Elemento & Concentração $(\mathrm{mg} / \mathrm{kg})$ \\
\hline $\mathrm{Fe}$ & 338 \\
\hline $\mathrm{Zn}$ & 225 \\
\hline $\mathrm{Cr}$ & 16,4 \\
\hline $\mathrm{Cu}$ & 0,2 \\
\hline $\mathrm{Ni}$ & 0,1 \\
\hline $\mathrm{Cd}$ & $<0,01$ \\
\hline
\end{tabular}

A disposição final da lama galvânica, seja em aterros industriais controlados (classe I) ou em processos de incineração, é onerosa. Há escassez de aterros classe I, que se encontram normalmente distantes dos polos produtores, acrescentado, assim, 0 custo de transporte. Além disso, há a responsabilidade perpétua pelo passivo ambiental de todo o resíduo descartado em aterros industriais. Diante de tal situação, algumas empresas deste setor optam por estocar seus resíduos enquanto aguardam uma solução que alie destinação final ecologicamente adequada e economicamente viável [4,7-11].

\section{TRATAMENTO DOS EFLUENTES DA GALVANIZAÇÃO}

O tratamento convencional dos efluentes gerados em operações de galvanização é a precipitação química. Caso haja a presença de cianeto e/ou cromo no banho, é

\footnotetext{
* Contribuição técnica ao $69^{\circ}$ Congresso Anual da ABM - Internacional e ao 14ํㅡㄹ ENEMET - Encontro Nacional de Estudantes de Engenharia Metalúrgica, de Materiais e de Minas, 21 a 25 de julho de 2014, São Paulo, SP, Brasil.
} 


\section{Tabela 2}

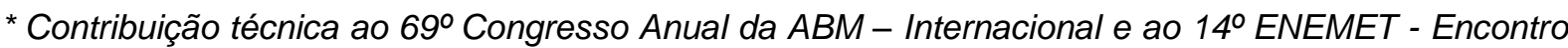
Nacional de Estudantes de Engenharia Metalúrgica, de Materiais e de Minas, 21 a 25 de julho de 2014, São Paulo, SP, Brasil. 


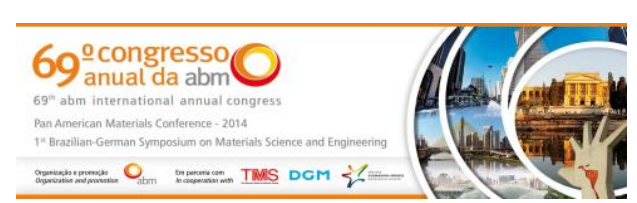

Tabela 2: Técnicas de separação propostas para o tratamento dos efluentes de galvanização $[2,4,5,10,16]$

\begin{tabular}{|c|c|c|c|}
\hline Processo & Aplicações & Vantagens & Desvantagens \\
\hline Troca iônica & $\begin{array}{c}\text { Baixa } \\
\text { concentração }\end{array}$ & $\begin{array}{c}\text { Remoção de constituintes } \\
\text { dissolvidos, fácil operação, } \\
\text { não geração de rejeitos, } \\
\text { possibilidade de reutilização } \\
\text { da água } \\
\end{array}$ & $\begin{array}{c}\text { Alto custo de investimento, de } \\
\text { operação e energia, e de difícil } \\
\text { manutenção }\end{array}$ \\
\hline Adsorção & $\begin{array}{c}\text { Baixa } \\
\text { concentração }\end{array}$ & $\begin{array}{l}\text { Remoção de constituintes } \\
\text { dissolvidos, fácil operação, } \\
\text { baixo custo de investimento } \\
\text { (exceto para resinas) }\end{array}$ & $\begin{array}{l}\text { Necessidade constante de } \\
\text { manutenção, susceptível à } \\
\text { geração de odor forte e } \\
\text { crescimento de bactérias }\end{array}$ \\
\hline $\begin{array}{l}\text { Osmose } \\
\text { reversa }\end{array}$ & $\begin{array}{c}\text { Baixa } \\
\text { concentração }\end{array}$ & $\begin{array}{c}\text { Remoção de constituintes } \\
\text { dissolvidos, fácil operação, } \\
\text { não geração de rejeitos, } \\
\text { possibilidade de reutilização } \\
\text { da água } \\
\end{array}$ & $\begin{array}{c}\text { Alto custo de investimento, de } \\
\text { operação e energia, e de difícil } \\
\text { manutenção }\end{array}$ \\
\hline $\begin{array}{l}\text { Extração por } \\
\text { solventes }\end{array}$ & $\begin{array}{c}\text { Alta } \\
\text { concentração }\end{array}$ & $\begin{array}{l}\text { Remoção de constituintes } \\
\text { dissolvidos, fácil operação, } \\
\text { elevada seletividade }\end{array}$ & $\begin{array}{l}\text { Emissões de voláteis, difícil } \\
\text { manutenção, geração de } \\
\text { efluentes que necessitam ser } \\
\text { tratados }\end{array}$ \\
\hline Eletrólise & $\begin{array}{c}\text { Alta } \\
\text { concentração }\end{array}$ & $\begin{array}{l}\text { Remoção de constituintes } \\
\text { dissolvidos, fácil operação, } \\
\text { não geração de rejeitos }\end{array}$ & $\begin{array}{l}\text { Alto custo de investimento, de } \\
\text { operação e energia, e de difícil } \\
\text { manutenção }\end{array}$ \\
\hline
\end{tabular}

Dentre os métodos listados na Tabela 2, a extração por solventes tem-se destacado devido à elevada seletividade obtida na separação dos metais presentes nos banhos. Vale destacar a recuperação seletiva do zinco utilizando-se fosfato de tri-n-butila (TBP) como extratante [4], e também com Cyanex 301, que apresentou excelente desempenho tanto em termos de percentual de extração de zinco quanto em termos de seletividade zinco-ferro [16,17]. Em relação aos metais cromo, níquel e cobre, destacam-se ainda os processos de adsorção e osmose reversa. Como exemplo, tem-se o uso de materiais de origem biológica (bactérias, fungos e algas) como adsorventes na remoção de diversos íons metálicos, processo conhecido por biossorção [18]. Apesar dos resultados já obtidos, há necessidade de mais estudos na área para que se possa encontrar um método que apresente melhor recuperação seletiva, além de ser econômico e ambientalmente adequado para cada situação particular.

\section{PROCESSOS DE ESTABILIZAÇÃO E REUTILIZAÇÃO}

Os processos de estabilização visam tornar inertes os elementos tóxicos contidos nos resíduos, de forma a transformá-los em estruturas químicas menos solúveis e/ou menos tóxicas. Tal transformação se dá por meio de reações químicas que fixam elementos ou compostos tóxicos tornando-os estáveis. Os processos de estabilização de resíduos consistem em reutiliza-los como matéria-prima em algum processo industrial, sendo assim, esses métodos constituem formas seguras e econômicas de descarte. No entanto, muitas vezes além de ser um descarte econômico, o emprego do resíduo acarreta em economia da matéria-prima empregada, além de benefícios nas características do material final.

Um exemplo de estabilização da lama galvânica é sua incorporação ao clínquer antes da calcinação na fabricação do cimento. O processo se mostra uma solução viável, uma vez que testes de lixiviação e de solubilidade comprovaram a

\footnotetext{
* Contribuição técnica ao 69ำ Congresso Anual da ABM - Internacional e ao 14ํㅡㄹ ENEMET - Encontro Nacional de Estudantes de Engenharia Metalúrgica, de Materiais e de Minas, 21 a 25 de julho de 2014, São Paulo, SP, Brasil.
} 


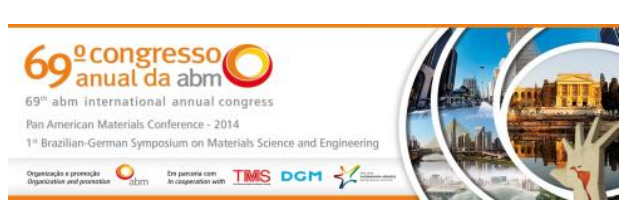

incapacidade dos metais aprisionados nas estruturas químicas do cimento de causarem problemas ambientais [12,15].

Outra alternativa é a incorporação da lama galvânica na formulação de pigmentos inorgânicos. O resíduo é incorporado ao banho de óxidos metálicos antes da etapa de calcinação, que após um ciclo de 8 horas formam fases estáveis sob elevadas temperaturas, provocando a estabilização dos metais da lama. Estudos revelaram que é possível incorporar até $20 \%$ do resíduo de galvanização na produção de pigmentos inorgânicos a base de ferro, cromo e zinco, sem que as características e qualidades fundamentais dos mesmos fossem alteradas [19].

Pode-se também adicionar a lama a materiais cerâmicos. Nesse caso, observou-se, através de técnicas de caracterização, a ocorrência de mudanças na ordem estrutural, em que os metais tornam-se aprisionados na microestrutura da cerâmica. Porém as propriedades técnicas das massas cerâmicas praticamente não foram alteradas em incorporações de até $2 \%$ de resíduo lavado [20]. De um modo geral, com relação às propriedades mecânicas da cerâmica, a avaliação se mostrou técnica e economicamente viável. Testes de lixiviação e solubilização comprovaram que os metais formam estruturas estáveis após a etapa de calcinação [15].

Há também a possibilidade de incorporação da lama na fabricação de fritas cerâmicas. Estes são materiais amorfos obtidos a partir de vidros, e constituem matéria-prima para se obter os esmaltes cerâmicos. Fritas com boa estabilidade química a partir de formulações contendo resíduos sólidos dos processos de galvanização foram obtidas [21]. A formulação de fritas com $23 \%$ em massa de resíduo sólido galvânico incorporado em uma matriz vítrea de composição comercial foi a que apresentou melhor poder de cobertura. Tal adição representa um percentual significativo em termos de integração de um resíduo em um produto.

A incorporação das lamas galvânicas nas emulsões asfálticas, além de estabilizar os elementos tóxicos, acarreta em uma melhor estabilização da matriz asfáltica, acelerando o processo de solidificação, conferindo maior estabilidade térmica e estrutural, melhorando o transporte e o armazenamento do asfalto [15].

E por fim, a lama pode ser reutilizada como um ativador das reações químicas durante a fabricação da borracha. Normalmente esses ativadores são óxidos metálicos, principalmente o óxido de zinco. Sendo assim, a incorporação dos resíduos da galvanização à borracha natural, em substituição parcial ou total ao óxido de zinco, permite dar um fim ecologicamente viável ao resíduo [15]. Porém, tal aplicação ainda encontra-se em fase de estudos e desenvolvimento.

\section{CONSIDERAÇÕES FINAIS}

A lama galvânica é um resíduo perigoso cuja quantidade gerada tende a aumentar dada a crescente necessidade de produção de aço galvanizado. Na Tabela 3 é apresentado um resumo das diversas formas de gerenciamento da lama galvânica apontadas no levantamento aqui realizado.

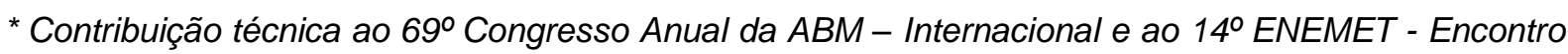
Nacional de Estudantes de Engenharia Metalúrgica, de Materiais e de Minas, 21 a 25 de julho de 2014, São Paulo, SP, Brasil.
} 


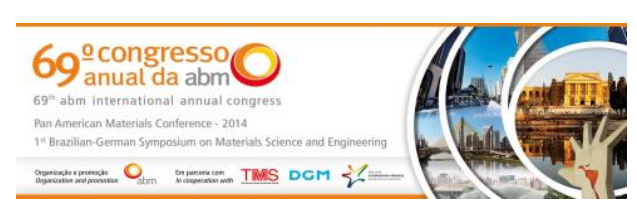

\section{Agradecimentos}

Os autores agradecem ao CNPq, CAPES, FAPEMIG e INCT-Acqua pelo apoio à pesquisa.

\section{REFERÊNCIAS}

1 Instituto de Metais Não Ferrosos - ICZ. Guia de galvanização por imersão a quente. Editado por: ICZ - Instituto de Metais Não Ferrosos, 2010 [acesso em: 19 jun. 2013]; 54p. Disponível em: <http://www.icz.org.br/portaldagalvanizacao/bibliotecapublicacoes.php>.

2 Rossini G. Emprego da sulfatação na recuperação de metais de lodos galvânicos [Dissertação de Mestrado]. Porto Alegre: Universidade Federal do Rio Grande do Sul, 2006.

3 Silva JE. Valorização de lamas galvânicas por via hidrometalúrgica [Tese de Doutorado]. Portugal: Universidade do Minho, 2006.

4 Pereira Neto A, Souza JB, Magalhães FS, Mansur MB, Rocha SDF. Alternativas para o tratamento de efluentes da indústria galvânica. Engenharia Sanitária e Ambiental, 2008; 13(3):263-270.

5 Simas R, Pawlowsky U. Recuperação de metais de resíduos galvânicos. São Paulo: 14 Encontro e Exposição Brasileira de Tratamento de Superfícies (EBRATS) - Catálogo oficial, 2012:152-159.

6 Associação Brasileira De Normas Técnicas. NBR 10004: Resíduos sólidos classificação. Rio de Janeiro: ABNT, 2004.

7 Ladeira ACQ, Pereira DBA. Avaliação do potencial poluidor da indústria galvânica: caracterização, classificação e destinação de resíduos. Revista Escola de Minas, Ouro Preto, 2008; 61(3):385-390.

8 Rossini G, Bernardes AM. Avaliação do tratamento pirometalúrgico da mistura de rejeito piritoso e lodo galvânico. Florianópolis: Congresso Brasileiro de Ciência e Tecnologia em Resíduos e Desenvolvimento Sustentável, 2004: 3050-3061.

9 Rossini G, Bernardes AM. Galvanic sludge metals recovery by pyrometallurgical and hydrometallurgical treatment. Journal of Hazardous Materials, 2006; 131(1-3):210-216.

10 Benedetto JS, Ladeira ACQ. Tratamento e reciclagem de efluentes líquidos da indústria metal-metalúrgica. Belo Horizonte: Relatório de projeto de pesquisa - Centro de Desenvolvimento da Tecnologia Nuclear - CDTN/CNEN, 2007. 88f.

11 Nolasco-Sobrinho, P.J.; Espinosa, D.C.R.; Tenório, J.A.S. Characterisation of dusts and sludges generated during stainless steel production in Brazilian industries. Ironmaking and Steelmaking, 2003; 30(1):11-17.

12 Villegas EA. Introdução ao controle ambiental na indústria metalúrgica. Belo Horizonte, Universidade Federal de Minas Gerais - Departamento de Engenharia Metalúrgica, 2009. 185p.

13 Piveli RP. Tratamento de efluentes de galvanoplastia. São Paulo: Anais da Conferência Internacional Sobre Prevenção e Controle da Poluição Industrial, 1993.

14 Riani JC. Utilização de resinas de troca-iônica em efluentes de galvanoplastia [Tese de Doutorado]. São Paulo: Escola Politécnica da Universidade de São Paulo, 2008.

15 Santos, A.C.S. Avaliação do efeito de resíduos galvânicos sobre a vulcanização e as propriedades da borracha natural [Dissertação de Mestrado]. Nova Friburgo: Universidade do Estado do Rio de Janeiro, 2009.

16 Hauers DSR, Castro FPM, Dorella G, Braga RC, Ricotta TQN, Hashizume TKC, Mansur $\mathrm{MB}$ et al. Estudo da separação zinco-ferro de efluente de galvanoplastia por extração líquido-líquido. Projeto de pesquisa - Universidade Federal de Minas Gerais, Belo Horizonte. 2006. $76 f$.

\footnotetext{
* Contribuição técnica ao $69^{\circ}$ Congresso Anual da ABM - Internacional e ao 14ํㅡㄹ ENEMET - Encontro Nacional de Estudantes de Engenharia Metalúrgica, de Materiais e de Minas, 21 a 25 de julho de 2014, São Paulo, SP, Brasil.
} 
17 Mansur MB, Rocha SDF, Magalhães FS, Benedetto JS. Selective extraction of zinc(II) over iron(II) from spent hydrochloric acid pickling effluents by liquid-liquid extraction. Journal of Hazardous Materials, 2008; 150:669-678.

18 Veit MT, Silva EA, Fagundes-Klen MR, Tavares CRG, Gonçalves GC. Biossorção de níquel e cromo de um efluente de galvanoplastia utilizando alga marinha pré-tratada em coluna. Acta Scientiarum Technology, 2009; 31(2):1175-183.

19 Milanez KW, Kniess CT, Bernardin AM, Riella HG, Kuhnen NC. Caracterização de pigmentos inorgânicos à base de $\mathrm{Fe}, \mathrm{Zn}$ e $\mathrm{Cr}$ utilizando resíduo de galvanoplastia como matéria-prima. Cerâmica, 2005; 51:107-110.

20 Balaton VT, Gonçalves PS, Ferrer LM. Incorporação de resíduos sólidos galvânicos em massas de cerâmica vermelha. Cerâmica Industrial, 2002; 7(6):42-45.

21 Marialva Neto AA. Incorporação de resíduos sólidos galvânicos em matrizes vítreas [Dissertação de Mestrado]. São Paulo: Instituto de Pesquisa Energéticas e Nucleares, 2003.

* Contribuição técnica ao $69^{\circ}$ Congresso Anual da ABM - Internacional e ao 14ํㅡㄹ ENEMET - Encontro Nacional de Estudantes de Engenharia Metalúrgica, de Materiais e de Minas, 21 a 25 de julho de 2014, São Paulo, SP, Brasil. 